\title{
MENSURAÇÃO DA APTIDÃO AERÓBIA ATRAVÉS DOS TESTES DE 12 MINUTOS E VELOCIDADE CRÍTICA APÓS OITO SEMANAS DE TREINAMENTO AERÓBIO EM MILITARES
}

\author{
Assesment of aerobic aptitude through 12 minute and critical velocity \\ tests after eight weeks of aerobic training in militaries
}

\author{
Wladimir Rafael Beck ${ }^{1}$, Alessandro Moura Zagatto¹. \\ ${ }^{1}$ Laboratório de Pesquisa em Fisiologia do Exercício - LAPEFE - UFMS - MS
}

\begin{abstract}
Resumo: A utilização de protocolos não invasivos para a avaliação e prescrição de exercícios tem sido muito importante nos últimos anos. No entanto, poucos são os estudos que analisam os efeitos de um treinamento sistematizado sobre as variáveis destes protocolos. Com isto, o objetivo deste estudo foi o de verificar o efeito do treinamento aeróbio de oito semanas na intensidade correspondente à velocidade crítica (VCrit) nas performances de 400, 800,1600 e 2800 metros e aptidão aeróbia, mensuradas pelos testes de 12 minutos e VCrit. Participaram do estudo 10 militares do sexo masculino,

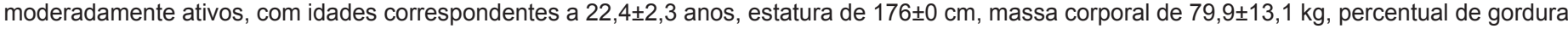
de $20,2 \pm 8,3 \%$, índice de massa corporal de $25,8 \pm 4,2 \mathrm{~kg} \cdot \mathrm{m}^{-2}$, e consumo máximo de oxigênio predito de $48,5 \pm 6,9 \mathrm{ml} \cdot \mathrm{kg}^{-1} \cdot \mathrm{min}^{-1}$. Todos foram submetidos ao teste de VCrit (cargas preditivas nas distâncias de 400, 800, 1600 e 2800 metros) e 12 minutos antes e após o treinamento. O treinamento consistiu em corrida com duração de 20 minutos por dia, três vezes por semana e intensidade correspondente à VCrit. Após o treinamento foram encontradas melhoras significativas nas intensidades correspondentes à VCrit, de $6 \%(p=0,03)$, assim como nas performances de 400, 1600 e $2800 \mathrm{~m}$, e na distancia total percorrida no teste de 12 minutos (D12min) após o treinamento, com um incremento nesta variável de 4,7\% ( $p=0,01$ ). Desse modo, podemos concluir que o treinamento aeróbio sistematizado de oito semanas na intensidade de VCrit, parece promover alterações significativas nesse parâmetro, assim como melhorar significativamente as performances de 400, 1600, 2800m e D12min.
\end{abstract}

Palavras Chave: Velocidade crítica, predição de performances, efeito de treinamento.

\begin{abstract}
The use of non-invasive protocols for prescription and evaluation of exercise has been much studied recently. However, few studies investigated the performance and physiological responses after a systematic training. Therefore, the purpose of the study was to verify the effects of eight weeks of aerobic training in critical velocity (VCrit), 12-minute test and performances in 400, 800, 1600 and 2.800 meters. Ten male militaries were participants of the study (age: $22.4 \pm 2.3$ years; body mass: $79.9 \pm 13.1 \mathrm{~kg}$; height: $176 \pm 0$ centimeters; body fat: $20.2 \pm 8.3 \%$; body mass index: $25.8 \pm 4.2 \mathrm{~kg}$. $\mathrm{m}^{-2}$; and predicted maximal oxygen uptake: $48.5 \pm 6.9 \mathrm{ml} . \mathrm{kg}^{-1} \cdot \mathrm{min}^{-1}$. All participants were submitted to VCrit test (predictive loads in $400,800,1600$ and 2800 meters distance) and 12-minute test before and after the training. The running training consists of 20 minutes duration per day, three times a week and intensity corresponding to VCrit. After the training, significant increases were found in the intensity corresponding to VCrit by $6 \%$ ( $p=0.03$ ), as well as in the performances of 400, 800 and 2800 meters, and also in distance of the 12-minute tests (D12min) (increase of $4.7 \%$; $p=0.009$ ). Therefore, the authors can conclude that the systematic aerobic training of eight weeks on VCrit intensity, seems to increase aerobic aptitude significantly as well as the performances in 400, 1600, 2800 meters and D12min.
\end{abstract}

Key words: Critical Velocity. Performances Prediction. Effect of the training

Aceito em 13/11/2008 - Rev. Educ. Fís. 2009 Mar: 144: 23-29. Rio de Janeiro - RJ - Brasil

\section{INTRODUÇÃO}

A utilização do lactato sanguíneo para mensurar a aptidão aeróbia tem obtido grande atenção nos últimos anos. Contudo, para a utilização de procedimentos que mensuram a aptidão aeróbia com o uso da concentração do lactato, torna-se necessário o uso de equipamentos sofisticados e diversos materiais de coleta sanguínea, o que acarreta em um alto custo. Além disso, a participação de pesquisadores experientes é imprescindível para a manutenção da fidedignidade do protocolo, tornando estes procedimentos de difícil acesso à academias, clubes e até mesmo para equipes desportivas com restrito recurso financeiro. No Caso do Exército Brasileiro, que avalia constantemente um número elevado de indivíduos, a aplicabilidade administrativa deste protocolo de 
determinação direta do limiar Anaeróbio (LAn) torna-se inviável. Por isso, recentemente, vários estudos têm procurado identificar procedimentos de avaliação que não apresentem alto custo financeiro, sejam não-invasivos e que possam determinar a aptidão aeróbia com confiabilidade, como é o caso de procedimentos que utilizam a concentração de lactato sanguíneo. Um destes procedimentos não invasivos que podem ser utilizados com certa facilidade é o modelo de potência crítica (PCrit) desenvolvido inicialmente por Monod e Scherrer (1), que corresponde à mais alta intensidade de exercício que, teoricamente, pode ser mantida por um período indefinido, sem que ocorra a exaustão. Esse modelo tem sido aplicado a eventos esportivos como corrida ${ }^{(2)}$, natação ${ }^{(3,4)}$, caiaque ${ }^{(5)}$, ciclismo ${ }^{(6)}$, tênis de mesa ${ }^{(7,8)}$ e tem apresentado boa correlação com o limiar anaeróbio ${ }^{(4)}$, com a performance aeróbia ${ }^{(9)}$, limiar anaeróbio individual (IAT) ${ }^{(10)}$ e com a máxima fase estável de lactato (MFEL) ${ }^{(11)}$. Kokubun ${ }^{(4)}$, Wakayoshi et al. (12) e Zagatto (13) verificaram que no exercício prolongado e de intensidade constante, correspondente a $100 \%$ do modelo de potência crítica, ocorre um equilíbrio dinâmico na concentração de lactato sanguíneo. Porém, com um pequeno aumento na intensidade do exercício esse equilíbrio da lactacidemia é perdido. Hill e Ferguson (14) também relataram semelhante comportamento no consumo de oxigênio $\left(\mathrm{VO}_{2}\right)$, e que em intensidades supra-VCrit ocorre um aumento gradativo no $\mathrm{VO}_{2}$ até alcançar o consumo máximo de oxigênio $\left(\mathrm{VO}_{2 \text { máx }}\right)$, indicando que a VCrit parece ser uma boa ferramenta para a avaliação da capacidade aeróbia. Contudo, a maioria dos estudos investigou o modelo de potência crítica como procedimento de avaliação, comparando os seus resultados com outros procedimentos de avaliação ${ }^{(11,15,16,17,18)}$. A resposta dessa variável ao efeito do treinamento ou destreinamento ainda não foi muito investigada. Denadai et al., (19), investigaram a VCrit para a determinação dos efeitos de um treinamento no limiar anaeróbio em corredores de endurance, concluindo que o seu uso pode ser dependente do treinamento realizado. Além disso poucos estudos tem sido desenvolvidos com a participação exclusiva de militares, que possuem rotinas semelhantes e necessidades físicas específicas devido sua atividade tão peculiar. Como a ferramenta de avaliação do parâmetro aeróbio do Exército Brasileiro é o teste de 12 minutos, sua utilização neste estudo tornase de grande importância.

Com isto, o objetivo do nosso estudo foi o de verificar efeito do treinamento aeróbio de oito semanas, de intensidade correspondente à velocidade crítica, nas performances de 400, 800 , 1600 e 2800 metros e aptidão aeróbia, mensuradas pelos testes de VCrit e 12 minutos.

\section{METODOLOGIA}

\section{Amostra}

Foram participantes do estudo 10 militares do sexo masculino, integrantes da $9^{a}$ Companhia de Guardas, Campo Grande-MS. Todos os participantes foram informados dos riscos dos procedimentos, tendo assinado um termo de consentimento livre e esclarecido, antes do início dos testes. O presente trabalho foi aprovado pelo Comitê de Ética em Pesquisa da Universidade Federal de Mato Grosso do Sul. Os dados antropométricos e de aptidão aeróbia estão apresentados na TABELA 1.

A determinação do consumo máximo de oxigênio foi feita através do teste de 12 minutos ${ }^{(20)}$, que será mais delineado a seguir. $O$ consumo máximo de oxigênio foi estimado utilizando a equação:

$\mathrm{VO}_{2 \text { máx }}=[$ distância total percorrida $(m)-504] / 45$.

\section{Procedimentos Experimentais}

Os participantes realizaram um aquecimento com duração de cinco minutos de corrida em 

Peso (Kg)
Altura (cm)
$\operatorname{IMC}\left(\mathrm{Kg} / \mathrm{m}^{2}\right)$
$\%$ Gordura
$\mathrm{VO}_{2 \text { máx }}$

$79,9 \pm 13,1$

$176 \pm 0,1$

$25,8 \pm 4,2$

$20,2 \pm 8,3$

$48,5 \pm 6,9$

intensidade moderada, controlada subjetivamente pelo participante. Todos os testes foram aplicados em pista de atletismo de 400 metros, no mesmo período do dia, com temperatura ambiente em aproximadamente $30^{\circ} \mathrm{C}$ e umidade relativa do ar de aproximadamente $40 \%$. Antes do treinamento, foi aplicado primeiramente o teste de 12 minutos, seguido pelo teste de VCrit. Este procedimento foi novamente realizado após o período de treinamento de oito semanas. Os testes foram aplicados com intervalo mínimo de 48 horas e finalizados em aproximadamente 10 dias em cada etapa. Durante todos os procedimentos os participantes foram estimulados verbalmente a realizarem a melhor performance possível.

\section{Determinação da Velocidade Crítica (VCrit)}

Para a determinação da velocidade crítica (VCrit) foram aplicadas quatro séries de exercício, nas distâncias fixas de 400, 800, 1600 e 2800 metros, sendo os participantes instruídos a realizarem essas distâncias no menor tempo possível. O tempo de exercício em cada distância foi registrado para posterior determinação da VCrit. Foram aplicadas no máximo duas séries de exercício por dia, segundo uma ordem aleatória, com intervalo de pelo menos duas horas entre os esforços. A VCrit foi determinada através da relação linear entre a distância e o tempo de corrida, sendo correspondente ao coeficiente angular da reta de regressão (FIGURA 1).
FIGURA 1.

RELAÇÃO LINEAR ENTRE A DISTÂNCIA PERCORRIDA E O TEMPO DE ESFORÇO PARA DETERMINAÇÃO DA VELOCIDADE CRÍTICA (VCrit), ANTES DO PERÍODO DE TREINAMENTO.

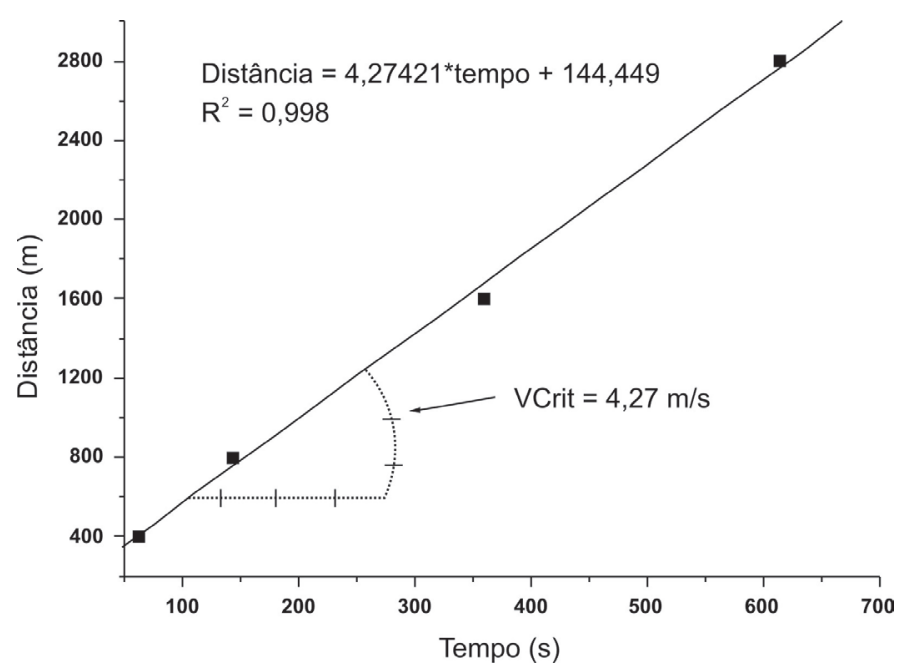

\section{Determinação da Distância Percorrida no Teste} de 12 Minutos

No teste de 12 minutos os avaliados foram instruídos a percorrer a maior distância possível no período de tempo pré-estipulado de 12 minutos ${ }^{(20)}$. O tempo decorrido foi anunciado aos nove, 11 e 11,5 minutos durante o teste através de sinal sonoro. A pista foi demarcada a cada 50 metros para facilitar a mensuração da distância percorrida. Após o término do teste (realizado com sinal sonoro) os participantes permaneceram no mesmo local até a mensuração da distância total percorrida. 


\section{Treinamento Físico}

O treinamento foi realizado de maneira sistematizada, com duração de oito semanas. A freqüência de treino foi de três sessões por semana, sendo a parte principal, corrida com duração de 20 minutos na intensidade correspondente a $100 \%$ da velocidade crítica determinada previamente.

\section{Procedimentos Estatísticos}

Os resultados obtidos foram expressos em média \pm desvio padrão. A normalidade das variáveis foi verificada pelo teste de Kolmogorov-Smirnov \& Liliefors e posteriormente adotada a estatística paramétrica. Foram também utilizados o teste " $\mathrm{t}$ " para amostras dependentes, nas comparações dos tempos obtidos nas distâncias de $400 \mathrm{~m}, 800 \mathrm{~m}$, $1600 \mathrm{~m}, 2800 \mathrm{~m}$ e VCrit, e também para a D12min, antes e após o treinamento. O teste de correlação produto-momento de Pearson foi utilizado para analise da associação das variáveis descritas acima antes e após o treinamento. Para todos os casos foi utilizado nível de significância de $5 \%$.

\section{RESULTADOS}

Todas as variáveis analisadas nesse estudo apresentaram distribuições normais quando verificadas pelo teste de normalidade de Kolmogorov-Smirnov \& Liliefors.

Para a determinação da velocidade crítica foram aplicadas quatro corridas máximas, nas distâncias de 400, 800, 1600 e 2800 metros, antes e após o treinamento, assim como foi avaliada a distância total percorrida no teste de 12 minutos, antes e após o treinamento. Os tempos de esforços em cada intensidade aplicada são apresentados na TABELA 2, e apenas os tempos de 800 m não apresentaram diferença significativa após o treinamento. Foram encontrados elevados valores de coeficiente de

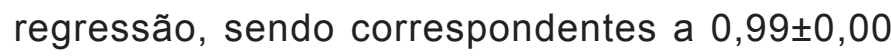
e 0,99 $\pm 0,00$, respectivamente, antes e após o treinamento. A capacidade de trabalho anaeróbia (CTA), que constitui o componente anaeróbio do protocolo de velocidade crítica, não apresentou

\section{TABELA 2.}

RESULTADOS DOS TEMPOS OBTIDOS PARA DETERMINAÇÃO DO TESTE DE VCrit, E DISTÂNCIA TOTAL PERCORRIDA NO TESTE DE 12 MINUTOS, PRÉ E PÓS-TREINAMENTO

\begin{tabular}{cccc}
\hline Variável & Pré & Pós & $P$-valor \\
\hline T400 (s) & $73,3 \pm 10,2$ & $68,1 \pm 7,1$ & $<0,01$ \\
T800 (s) & $174,3 \pm 26,0$ & $171,0 \pm 24,3$ & 0,30 \\
T1600 (s) & $427,5 \pm 42,5$ & $396,5 \pm 46,3$ & 0,01 \\
T2800 (s) & $799,8 \pm 117,0$ & $756,7 \pm 97,2$ & 0,02 \\
\hline T12 $\min (\mathbf{m})$ & $2688,6 \pm 313,6$ & $2808,7 \pm 285,3$ & $<0,01$ \\
\hline
\end{tabular}


27 Rev. Educ. Fís. 2009 Mar: 144: 23-29. Rio de Janeiro - RJ - Brasil

diferença significativa quando comparada nos diferentes períodos $(p=0,811)$.

As distâncias percorridas no teste de 12 minutos antes e após o treinamento foram significativamente diferentes, apresentando um percentual de melhora correspondente a $4,7 \%$. Os dados relativos à VCrit antes e após o treinamento estão representadas na FIGURA 2.

FIGURA 2.

RESULTADO DA MÉDIA ENCONTRADA NO TESTE DE VCrit ENTRE TODOS OS PARTICIPANTES ANTES E APÓS O TREINAMENTO DE OITO SEMANAS.

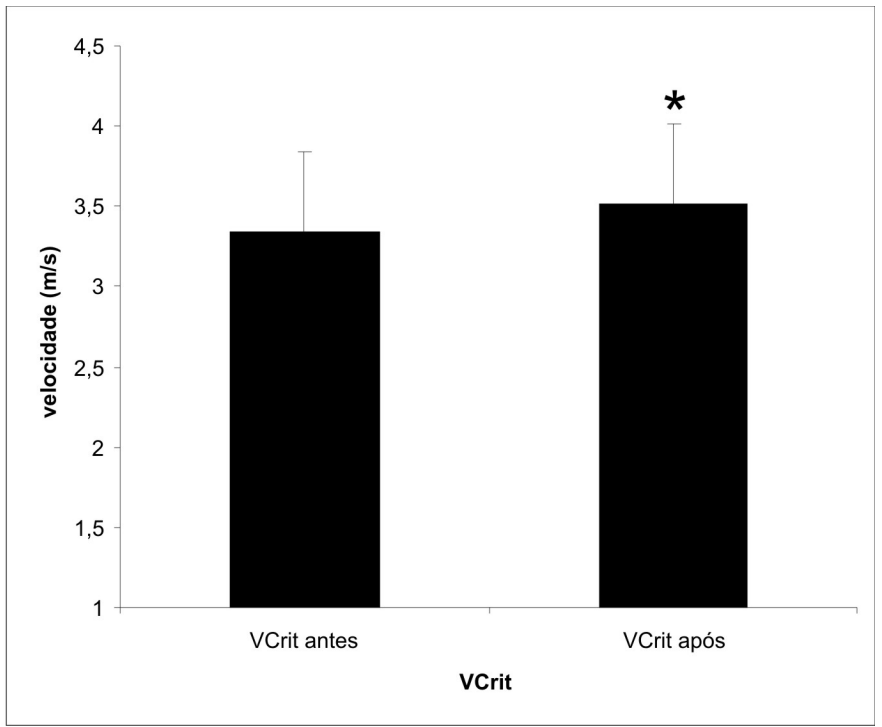

${ }^{*} p<0,05$ em relação à VCrit antes.

Após o treinamento, a intensidade de velocidade crítica apresentou um percentual de melhora correspondente a $6 \%$, comparativamente com o valor pré-treinamento. A velocidade crítica também mostrou ser boa preditora de performances apresentando significativas correlações com as performances de 400, 800, 1600 e 2800 metros e também com a distância total percorrida no teste de 12 minutos, tanto quando comparados os resultados antes do treinamento quanto após o treinamento de oito semanas (TABELA 3).
TABELA 3.

RESULTADOS DO TESTE DE CORRELAÇÃO DE PEARSON ENTRE AS VARIÁVEIS, ANTES E APÓS O PERÍODO DE TREINAMENTO

\begin{tabular}{ccc}
\hline & \multicolumn{2}{c}{ VCrit } \\
\cline { 2 - 3 } & Antes & Depois \\
\hline D12min & $0,93^{*}$ & $0,96^{*}$ \\
T400 & $-0,85^{*}$ & $-0,97^{*}$ \\
T800 & $-0,89^{*}$ & $-0,95^{*}$ \\
T1600 & $-0,93^{*}$ & $-0,98^{*}$ \\
T2800 & $-0,98^{*}$ & $-0,99^{*}$ \\
\hline
\end{tabular}

${ }^{*} \mathrm{P}<0,05$ considerado significante.

\section{DISCUSSÃO}

Os principais achados desse estudo foram as melhoras significativas nas intensidades correspondentes à velocidade crítica, nas performances de 400, 1600, 2800 e na distância percorrida no teste de 12 minutos após o treinamento de oito semanas (de 4,7\%), e também as correlações significativas da VCrit e com todas as performances tanto antes como após o treinamento.

A potência crítica e suas adaptações (velocidade critica, freqüência crítica, força crítica) parecem ser boas ferramentas para a predição de performances de longa duração e também predição da intensidade correspondente à capacidade aeróbia ${ }^{211,10,22,4,23,11,24)}$. Apesar do protocolo de potência crítica ser muito estudado em relação a sua aplicação como preditor da intensidade de máxima fase estável de lactato e também como preditor da performance esportiva, poucos estudos investigaram o efeito do treinamento nessa variável $(6,25)$. Como a intensidade correspondente à VCrit tem sido relacionada com o limiar anaeróbio e parece representar o mesmo fenômeno, torna-se uma boa ferramenta para a prescrição de treinamento, principalmente o treinamento aeróbio. 
Dentre as poucas investigações sobre o efeito do treinamento nesse procedimento de avaliação, Jenkins e Quigley ${ }^{(26)}$ relataram que a potência critica parece aumentar com o treinamento físico sistematizado em intensidades prescritas a partir deste índice. Em um treinamento de oito semanas em cicloergômetro, com 30-40 minutos por dia, sendo três vezes por semana na intensidade correspondente à potência crítica foi encontrada melhora significativa no VO2máx $(8,5 \%)$ e na potência crítica (31\%). Kokubun (4) investigou nadadores e analisou a reposta da VCrit determinada no período específico e também no período competitivo, e encontrou melhora significativa nessa variável com a mudança da fase de treinamento. Em nosso estudo prescrevemos o exercício durante oito semanas, com duração diária de 20 minutos e freqüência semanal de três dias/semana, com a intensidade correspondente a $100 \%$ da VCrit e pudemos verificar aumento de $6 \%$ na intensidade correspondente a velocidade crítica, encontrando ainda diferença significativa entre antes e após, assim como relatado pelos mencionados anteriormente. O percentual de melhora da VCrit em nosso estudo foi menor que o relatado por Jenkins e Quigley ${ }^{26)}$. Isto pode ter ocorrido pelo nível de treinamento dos nossos participantes, que apesar de não serem atletas, apresentam um bom nível de aptidão física $\left(\mathrm{VO}_{2 \text { máx }}=48,5 \pm 6,9 \mathrm{ml} \cdot \mathrm{kg}-1 . \mathrm{min}-1\right)$ além de a duração do treinamento ter sido menor que a prescrita por aqueles autores. Outra modificação importante verificada no estudo foi a melhora significativa nas performances de 400, 1600 e 2800 metros, assim como na D12min. Estranhamente não foi encontrada melhora na performance de $800 \mathrm{~m}$, apenas. Um dos motivos que poderia buscar uma explicação para esse resultado seria o fator motivacional, verificado e observado subjetivamente.

A intensidade correspondente à capacidade aeróbia (limiar anaeróbio, lactato mínimo, máxima fase estável de lactato, potência crítica) tem mostrado ser uma boa ferramenta de predição de performances, principalmente em provas esportivas com maior predominância aeróbia. Papoti et al. (23) também relataram que a velocidade crítica é uma boa preditora da performance de $400 \mathrm{~m}$. Hughson et al. ${ }^{(27)}$ encontraram resultado semelhante na corrida obtendo correlação entre a VCrit e a performance de $10 \mathrm{Km}$. Em nosso estudo foram verificadas altas e significativas correlações entre a VCrit e as performances, constatando que essa parâmetro parece ser um bom preditor de performance, principalmente em provas de longa duração, onde apresenta uma contribuição significativa do componente aeróbio.

\section{CONCLUSÃO}

A partir do apresentado, podemos concluir que o treinamento aeróbio sistematizado de oito semanas na intensidade de VCrit, parece promover alterações significativas nesse parâmetro, assim como melhorar significativamente as performances de 400, 1600, 2800m. D12min parece melhorar após o treinamento proposto quando avaliada através do teste de 12 minutos e ainda, que a VCrit e D12min parecem ser ótimas preditoras de performances em corrida.

\section{REFERÊNCIAS BIBLIOGRÁFICAS}

1. Monod H, Scherrer J. The Work Capacity of a synergic muscular group. Ergonomics 1965;8:329-38.

2. Bosquet L, Duchene A, Lecot F, Dupont G, Leger L. $\checkmark$ max estimate from three-parameter critical velocity models: validity and impact on $800 \mathrm{~m}$ running performance prediction. Eur J Appl Physiol 2006;97(1):34-42.

3. Papoti M. Efeitos do polimento sobre performances aeróbia e anaeróbia de nadadores após ciclo experimental de treinamento. Dissertação de Mestrado. Rio Claro-SP: Universidade Estadual Paulista, UNESP 2003.

4. Kokubun, E. Velocidade crítica como estimador do Limiar Anaeróbio na natação. Revista Paulista de Educação Física 1996;1:5-20.

5. Clingeleffer A, Mcnaughton LR, Davoren B. The use of critical power as a determinant for establishing the onset of blood lactate accumulation. Eur J Appl Physiol 1994;68:182-7. 
29 Rev. Educ. Fís. 2009 Mar: 144: 23-29. Rio de Janeiro - RJ - Brasil

6. Jenkins GD, Quigley BM. The influence of high-intensity exercise training on the Wlim-Tlim relationship. Med Sci Sports Exerc 1993;24:1283-9.

7. Zagatto AM, Gobatto CA. Determinação de um modelo de avaliação aeróbia no tênis de mesa em protocolo específico utilizando robô. Table Tennis Players 2002;15:10-11.

8. Zagatto AM, Gobatto CA. Validação do modelo de frequência crítica em protocolo específico através de método indireto para o tênis de mesa. Lecturas Educación Física y Deportes 2007;110:1-7.

9. Morton HR, Billat LV. The critical power model for intermittent exercise. Eur J Appl Physiol 2004;91:303-7.

10. Mcllean TM, Cheung KSY. A comparative evalution of the individual anaerobic thresold and the critical power. Med Sci Sports Exerc 1992;24:543-50.

11. D’Angelo R, Gobatto CA. Predição da intensidade de corrida em máxima fase estável de lactato a partir da velocidade crítica em atletas fundistas de alto rendimento. Revista Brasileira de Ciência e Movimento 2006;14:50.

12. Wakayoshi K, Ikuta K, Yoshida T, Udo M, Moritani T, Mutoh Y, Miyashita M. Determination and validity of critical velocity as an index of swimming performance in the competitive swimmer. Eur J Appl Physiol 1992;64:153-7.

13. Zagatto AM. Determinação e validação da freqüência crítica e da capacidade de trabalho anaeróbio no tênis de mesa em protocolo específico utilizando robô. Dissertação de Mestrado. Rio Claro-SP: Universidade Estadual Paulista, UNESP 2004.

14. Hill DW, Ferguson CS. A physiological description of critical velocity. Eur J Appl Physiol 1999;79:290-3.

15. Colantonio, E. Analise das velocidades: referencial de $4 \mathrm{mM}$, de equilíbrio de 30 min e velocidade critica em nadadoras adolescentes. Dissertação de mestrado. São Paulo: Universidade de São Paulo, USP 1999:155.

16. Simões HG, Silva LGM, Pacheco ME, Campbell CSG, Baldissera V. Comparação entre protocolos diretos e indiretos de avaliação da aptidão aeróbia em indivíduos fisicamente ativos. Revista Brasileira de Medicina do Esporte 2005;11: 219-23.

17. Silva ASR, Santos FNC, Santhiago V, Gobatto CA. Comparação entre métodos invasivos e não invasivos de determinação da capacidade aeróbia em futebolistas profissionais. Revista Brasileira de Medicina do Esporte 2005;11:233-37.
18. Ferrari HG, Guglielmo LGA, Nascimento WT. Utilização da velocidade critica para predição do limiar anaeróbio na corrida. Revista Brasileira de Ciência e Movimento 2006;14:48.

19. Denadai BS, Ortiz MJ, Mello MT. Validade da velocidade critica para a determinação dos efeitos do treinamento no limiar anaeróbio em corredores de enducance. Revista Portuguesa de Ciências do Desporto 2003;3:16-23.

20. Cooper HK. A means of assessing maximal oxygen intake. JAMA 1968;203:135-8.

21. Moritani T, Nagata A, deVries HA, Muro M. Critical power as a mearure of physical work capacity and anaerobi thresould. Ergonomics 1981; 24:339-350.

22. Wakayoshi K, Takayoshi Y, Masao U, Takashi H, Toshio M, Yoshiteru M, Mitsumasa M. Does critical swimming velocity represents exercise intensity at maximal lactate steady state?. Eur J Appl Phisyol 1993; 66:90-95.

23. Papoti M, Zagatto AM, Freitas Júnior PB, Cunha AS, Martins LEB, Gobatto CA. Utilização do intercepto-y na avaliação da aptidão anaeróbia e predição da performance de nadadores treinados. Revista Brasileira de Medicina do Esporte 2005;11:126-30.

24. Zagatto AM, Papoti M, Gobatto CA. Validity of critical frequency test for measuring table tennis aerobic endurance through specific protocol. J Sports Sci Med 2008; in-press.

25. Bishop DG, Jenkins AH. The critical power is dependent on the duration of the predictive exercise tests chosen. Int J Sports Med 1996;19:125-9.

26. Jenkins GD, Quigley BM. Endurance training enhances critical power. Med Sci Sports Exerc 1992;24:1283-9.

27. Hughson RL, Orok CJ, Staud LE. A high velocity treadmill running test to assess endurance running potential. Int J Sports Med 1984;5:23-5.

Endereço:

Universidade Federal de Mato Grosso do Sul, Departamento de Educação Física. Av. Costa e Silva s/n. Cidade Universitária, Campo Grande/MS, Brasil.

CEP: 79070-900

e-mail: azagatto@yahoo.com.br ; ten_beck@ig.com.br 